\title{
Discovery of a MUC3B gene reconstructs the membrane mucin gene cluster on human
} chromosome 7

Tiange Lang ${ }^{1}$, Thaher Pelaseyed ${ }^{2,3, *}$

1. Big Data Decision Institution, Jinan University, Tianhe, Guangzhou, China.

2. Department of Medical Biochemistry and Cell Biology, Institute of Biomedicine, University of Gothenburg, Box 440, 40530 Gothenburg, Sweden.

3. Lead contact

* Corresponding author: thaher.pelaseyed@medkem.gu.se 


\begin{abstract}
Human tissue surfaces are coated with mucins, a family of macromolecular sugar-laden proteins serving diverse functions from lubrication to formation of selective biochemical barriers against harmful microorganisms and molecules. Membrane mucins are a distinct group of mucins that are attached to epithelial cell surfaces where they create a dense glycocalyx facing the extracellular environment. All mucin proteins carry long stretches of tandemly repeated sequences that undergo extensive O-linked glycosylation to form linear mucin domains. However, the repetitive nature of mucin domains makes them prone to recombination and render their genetic sequences particularly difficult to read with standard sequencing technologies. As a result, human mucin genes suffer from significant sequence gaps that have hampered investigation of gene function in health and disease. Here we leveraged a recent human genome assembly to identify a previously unmapped MUC3B gene located within a cluster of four structurally related membrane mucin genes that we entitle the MUC3 cluster at q22 locus in chromosome 7. We found that MUC3B shares high sequence identity with the known MUC3A gene, and that the two genes are governed by evolutionarily conserved regulatory elements. Furthermore, we show that MUC3A, MUC3B, MUC12 and MUC17 in the human MUC3 cluster are exclusively expressed in intestinal epithelial cells. Our results complete existing genetic gaps in the MUC3 cluster that is a conserved genetic unit during primate evolution. We anticipate our results to be the starting point for detection of new polymorphisms in the MUC3 cluster associated with human diseases. Moreover, our study provides the basis for exploration of intestinal mucin gene function in widely used experimental models such as human intestinal organoids and genetic mouse models.
\end{abstract}




\section{Introduction}

The first draft of the human genome published twenty years ago offered a unique opportunity to decipher the causal relationship between gene sequence, gene function and disease biology (Craig Venter et al., 2001; Lander et al., 2001). But reading and measuring repetitive genomic elements has remained a major technological challenge that has left the human genome riddled with significant sequence gaps. Mucin $(M U C)$ genes are characterized by subexonic repeats, characterized by multiple repeated short DNA sequences within coding exons. The resulting tandemly repeated sequences encode long domains rich in proline, threonine and serine (PTS) residues (Lang et al., 2007). Mucin-type tandem repeats undergo O-linked glycosylation on serines and threonines to form densely O-glycosylated linear mucin domains (Nason et al., 2021). The number of tandem repeats and their sequence identity vary between MUC genes and are further confounded by considerable length polymorphism between individuals, resulting in variable number tandem repeats (VNTRs). However, VNTRs present a major challenge in analyzing mucin sequences as their repetitive nature and size in several kilobases cause intrinsic instabilities that make them difficult to maintain in bacterial artificial chromosome clones. Consequently, mucin gene VNTRs are underrepresented in human genome assemblies (Chaisson et al., 2015) and continue to hamper efforts to investigation of $M U C$ gene function.

Mucins are an ancient family of proteins in the animal kingdom. The earliest mucin genes appeared 700-800 million years ago in primitive marine metazoans such as sea anemones, sponges and jelly combs and have since expanded to all branches of the tree of life (Hedges et al., 2006; Lang et al., 2016). Currently, the human mucin family consists of secreted gelforming mucins (MUC2, MUC5A, MUC5B, MUC5AC, MUC6, MUC7 and MUC20) and a distinct subfamily of membrane mucins (MUC1, MUC3, MUC4, MUC12, MUC13, MUC15, MUC16, MUC17, MUC21 and MUC22) that are inserted into cell membranes via a transmembrane domain (Pelaseyed and Hansson, 2020). Membrane mucins are single-pass type I transmembrane proteins that are guided to the secretory pathway via an N-terminal signal sequence. In the endoplasmic reticulum, membrane mucins undergoes $\mathrm{N}$-linked glycosylation and folding, which in most cases requires a strain-dependent autocatalytic cleavage at a Sea urchin sperm protein, Enterokinase and Agrin (SEA) domain (Ligtenberg et al., 1992). The cleaved protein fragments remains non-covalently attached at the SEA domain as the mucin protein transits to the Golgi apparatus for O-linked glycosylation. Consequently, the mature SEA-type membrane mucin reach the plasma membrane as heterodimer of a glycosylated extracellular $\mathrm{N}$-terminal subunit that remains non-covalently linked to a membrane-attached C-terminal subunit. The evolutionary origins of the SEA domain date back to single-celled eukaryotes while SEA-type membrane mucins emerged in vertebrates (Lang 
et al., 2007; Pei and Grishin, 2017). While our earlier work has shown that the SEA domain undergoes conformational unfolding in response to mechanical tension, its ultimate function remains elusive (Pelaseyed et al., 2013).

In humans, membrane mucins genes MUC3, MUC12 and MUC17 map to the chromosomal locus 7q22. The three genes are arranged in a MUC3-MUC12-MUC17 cluster (hereafter called MUC3 cluster), and flanked by ACHE upstream of MUC3, and TRIM56 and SERPINE1 downstream of MUC17. The stereotypic ACHE-MUC3-MUC12-MUC17-TRIM56- SERPINE1 unit is highly conserved in vertebrates. Mus musculus carries a MUC3 cluster on chromosome 5 where three membrane mucin genes are flanked by Ache and Trim56-Serpine1. Notably, the Muc3 gene in M. musculus maps directly upstream of Trim56 and shares $43 \%$ sequence identity with human MUC17, but only $28 \%$ identity with human MUC3, indicating that murine Muc3 is a homolog of human MUC17 while the murine homologues for MUC3 and MUC12 are poorly defined. The nonmammalian vertebrate Xenopus tropicalis carries seven genes encoding SEA-type membrane mucins, of which three are arranged in tandem on chromosome 3 followed by a homolog of human SERPINE1, suggesting that the MUC3 cluster first emerged in amphibians (Lang et al., 2007).

The current human genome assembly (GRCh38.p13) is estimated to contain unsolved gaps corresponding to nearly 150 million base pairs (Mbp) (Nurk et al., 2021), which we postulate underlie the lack of complete sequences for human MUC genes in general and the MUC3 cluster in particular. In this work, we take advantage of the most recent T2T-CHM13 assembly of the human genome (Nurk et al., 2021) to provide evidence for the existence of a MUC3B genes in the human genome. We also demonstrate that MUC3A and MUC3B genes are conserved in late hominoids such as the chimpanzee as well as Old World monkeys. Finally, by exploring published RNA-sequencing data sets, and applying quantitative gene expression analysis in human tissues, we show that MUC $3 A$ and MUC3B expression is limited to intestinal epithelial cells.

\section{Results}

\section{The evolution of a MUC3 cluster in Cercopithecoids and Hominoids}

The human chromosome locus 7q22 contains three MUC genes MUC3, MUC12 and MUC17, arranged in a MUC3 cluster flanked by ACHE at its 5' end, and TRIM56 and SERPINE1 at its 3 ' end (Figure 1A). Using ACHE, TRIM56 and SERPINE1 as genomic markers, we identified the MUC3 cluster in species belonging to the Catarrhini parvorder, namely cercopithecoid (Old World monkeys) and hominoid superfamilies, the latter including the genera Pongo (orangutang), Gorilla, Pan (chimpanzee and bonobo) and Homo (Figure 1B). In cercopithecoids we identified a MUC3 cluster with a length of 153 kilo base pairs (kbp) in 
rhesus (Macaca mulatta), while the corresponding gene cluster in the baboon (Papio Anubis) consisted of two mapped sequences with a total length of $138 \mathrm{kbp}$ (Figure 1B). In hominoids, MUC3 cluster length ranged from $106 \mathrm{kbp}$ in the gibbon (Nomascus leucogenys) to $203 \mathrm{kbp}$ in the orangutang (Pongo abelii). In the Homininae subfamily, we observed striking differences between the MUC3 cluster length in chimpanzee (Pan troglodytes) and its two closest relatives; the gene cluster in $H$. sapiens GRCh38 assembly was $73 \mathrm{kbp}$ shorter and, in the gorilla (G. gorilla) $66 \mathrm{kbp}$ shorter, than in the chimpanzee (Figure 1B). Thus, we hypothesized that the current human GRCh38.p13 assembly contains significant gaps within the MUC3 cluster.

To test our hypothesis, we used a set of defined criteria for exploring distinct MUC genes within MUC3 clusters in available primate genome assemblies. We scanned the clusters for 1) start codons, 2) long mucin-type PTS-encoding exons, 3) conserved SEA domains specific for membrane mucins and, 4) unique intronic and exonic sequences that separate individual MUC genes. Our analysis revealed that all cercopithecoids carried a MUC3 cluster consisting of MUC3, MUC12 and MUC17 genes (Figure 1B). Strikingly, the primate MUC3 gene existed as two distinct $M U C 3 A$ and $M U C 3 B$ genes, although only partial sequences of the $\mathrm{N}$ - and $\mathrm{C}$ terminal fragments of a MUC3B gene were identified in $P$. anubis. The hominoid superfamily, with the exception of $H$. sapiens and G. gorilla, carried a MUC3A gene and full or partial sequences of MUC3B. By exploring all existing primate sequences flanked by ACHE, TRIM56 and SERPINE1, we concluded that a MUC3B gene emerged exclusively in the Catarrhini parvorder 29.4 million years ago (Mya) (Figure 1C).

\section{The human $7 q 22$ locus contains a MUC3B gene}

As humans and chimpanzees share $98.8 \%$ of their genomic DNA and the chimpanzee genome carries a MUC3B gene in the MUC3 cluster, we hypothesized that absence of a MUC3B gene at the human $7 q 22$ locus is a result of sequence gaps in the publicly available genome assembly. In the quest of a human MUC3B gene, we explored PacBio Single Molecule Real-Time (SMRT) reads from a human HX1 (Shi et al., 2016) and identified 3 individual reads that covered the 3 ' end region of $M U C 3 A$ (encoding the C-terminal region of MUC3A protein, designated MUC3A C-term), an intergenic region and the 5' end region of a putative MUC3B gene (designated MUC3B N-term) (Figure 2A). Strikingly, the length of intergenic region was in average $10,939 \mathrm{bp}$, which corresponded to the length of MUC3AMUC3B intergenic region in catarrhines (average 11,810 bp). Moreover, we identified 5 SMRT reads covering MUC3B C-term, an intergenic region and MUC12 N-term (Figure 2A). The average length of MUC3B-MUC12 intergenic region was 2469 bp and conserved in catarrhines (average $2491 \mathrm{bp}$ ). This initial exploration provided evidence for the existence of a distinct human MUC3B gene. However, because MUC3A and MUC3B share high sequence 
identity ( $87 \%$ and $94 \%$ for $\mathrm{N}$-term and C-term across catarrhines) and the error rate of the SMRT reads was $70-85 \%$, the $\mathrm{HX} 1$ assembly could not with high confidence distinguish between the two MUC3 genes. Moreover, the reads failed to capture the length and sequence identity of a predicted single PTS-encoding exon in MUC3B.

The current GRCh38.p13 draft covers lightly packed euchromatic regions corresponding to $92 \%$ of the human genome, while more complex regions including long tandems repeats in MUC genes are underrepresented. A recently published CHM13 T2T v1.1 assembly, based on long-read genome sequencing of homozygous complete hydatidiform mole (CHM) cells followed by gapless telomere-to-telomere assembly, adds approximately $200 \mathrm{Mbp}$ to the GRCh38.p13 assembly (Nurk et al., 2021). Importantly, the T2T-CHM13 assembly revealed a $60 \mathrm{kbp}$ gap between MUC3A and MUC12 at locus 7q22 (Figure 2B). Within this gap, we identified a 39,267 bp long PTS-encoding exon flanked upstream by a 2,187 bp long sequence with $87 \%$ identity to MUC3A N-term. Downstream of the PTS-encoding exon, we identified a 6,303 bp long sequence that was $92 \%$ identical with MUC3A C-term, and contained a SEA domain, a transmembrane domain and a cytoplasmic tail with a conserved PDZ motif (Malmberg et al., 2008) (Figure 2C). This data suggests that the T2T-CHM13 assembly contains a putative $M U C 3 B$ gene at locus $7 q 22$ with high sequence identity with the annotated MUC3A gene.

\section{Distinct human MUC3A and MUC3B genes share high sequence homology}

To better characterize a putative human $M U C 3 B$ gene, we compared the exon-intron structure of the new MUC3B gene to MUC3A. A previous study reported 11 exons in a putative MUC3B gene (Pratt et al., 2000). Our analysis of T2T-CHM13 assembly revealed that MUC3A and MUC3B both have 12 exons with near identical nucleotide lengths, with the exception of the PTS-encoding exon 2 that is 15,873 bp (5,291 amino acids) in MUC3A and 39,267 bp (13,089 amino acids) in $M U C 3 B$ (Figure $3 A$ ). Nucleotide sequence identity amongst individual exons of MUC3A and MUC3B was in average $93 \%$, and $92 \%$ for introns. The superfamilies of hominoids (apes and humans) and cercopithecoids (Old World monkeys) diverged around 29 Mya (Hedges et al., 2006). Sequence alignments between $\mathrm{N}$-terminal and C-terminal of MUC genes in the MUC3 cluster showed a high degree of conservation between $H$. sapiens and members of the cercopithecoid and hominoid branches. Human MUC3A N-term was $99 \%$ identical to chimpanzee MUC3A N-term and $90-91 \%$ identical to MUC3A N-term in the cercopithecoid members rhesus and baboon. MUC3A C-term showed a slightly higher degree of divergence compared to MUC3A N-term (Figure 3B, Supplementary table 1). The same trend was observed for $M U C 3 B$, in which the $M U C 3 B$ C-term was less conserved that $M U C 3 B$ $\mathrm{N}$-term within the hominoid and cercopithecoid superfamilies. Tandem repeat regions are prone to duplications and deletions caused by recombination (Svensson et al., 2018). 
Accordingly, we observed higher evolutionary sequence divergence in the PTS-encoding exon 2 of MUC genes in the MUC3 cluster (Figure 3B, Supplementary table 1). Moreover, pairwise alignment of Catarrhini MUC3 cluster genes revealed a general trend towards expansion of tandem repeats during primate evolution (Figure S1). Specifically within exon 2 of human MUC3A and $M U C 3 B$, we identified imperfect repeats with $87 \%$ amino acid sequence identity between MUC3A and MUC3B. In addition, MUC3B contained an additional 1368 amino acids of imperfect repeats (Figure 3C). MUC3A and MUC3B also harbored 166 and 549 perfect tandem repeats of a 17 amino acids long consensus sequence (ITTTETTSHSTPSFTSS) (Figure 3D). Thus, we conclude that the genetic structure of MUC3B is highly similar to MUC3A and that the two MUC3 genes are likely paralogous genes characterized by variable number of tandem repeats.

\section{MUC3A and MUC3B are regulated by conserved regulatory elements}

Sequences upstream of a gene's transcription start site (TSS) contain regulatory elements that dictate gene expression, yet the regulatory elements for MUC3A and MUC3B genes have not been characterized. Sequence analysis of presumed regulatory sequences $-1 \mathrm{kbp}$ of MUC3A TSS identified a candidate cis-Regulatory Element (CCRE) at position -403 bp (Figure $4 \mathrm{~A}$ ) that shared $83 \%$ identity with the corresponding region in MUC3B. Published DNase I hypersensitive site sequencing (DNase-seq) data sets from human small intestine and colon revealed high DNase hypersensitivity signals within the cCRE (Figure 4A). Moreover, we identified high signals for active chromatin markers $\mathrm{H} 3 \mathrm{~K} 9 \mathrm{ac}$ and $\mathrm{H} 3 \mathrm{~K} 4 \mathrm{me} 3$ within the MUC3A cCRE in human small intestine and colon, while active chromatin signals in the stomach were either not detected or low. By predicting transcription factor binding sites (TFBSs) using the JASPAR CORE vertebrate collection (Fornes et al., 2020; Sandelin et al., 2004), we identified putative TFBSs in CCRE of MUC3A (Figure S2). Seven of these transcription factors (ELF3, HNF4A, HNF4G, KLF4, PPARA, STAT3 and XBP1) are enriched in transporting intestinal epithelial cells in human and mouse intestine (Figure 4B, Figure S3). An alignment of putative promoter regions upstream of $M U C 3 A$ and $M U C 3 B$ genes in hominoid and cercopithecoid superfamilies identified conserved TFBSs for HNF4A, HNF4G and STAT3, strongly suggesting that the two MUC3 genes share an evolutionarily conserved regulatory expression program in the small intestine and colon (Figure 4C, Figure S4, Supplementary table 2).

\section{Expression of human MUC3A and MUC3B genes in the human intestine}

To determine whether MUC3B is transcribed into messenger RNA, we mapped published RNA-sequencing data sets from human intestine (Wang et al., 2020), liver (Aizarani et al., 2019) and kidney (Su et al., 2021) to the T2T-CHM13 assembly. A considerable number of sequenced reads from $M U C 3 A$ and $M U C 3 A$ transcripts were detected in human ileum, colon 
and rectum, while liver and kidney were devoid of transcripts from MUC3 cluster genes (Supplementary table 3). Because the exons encoding the $\mathrm{N}$-terminal, PTS and C-terminal regions of $M U C 3 A$ and $M U C 3 B$ share $87 \%, 83 \%$ and $92 \%$ identity and PTS-encoding exons are highly repetitive, we predicted a challenge in detecting sufficient unique sequencing information to accurately assign reads to the correct MUC3 gene. Therefore, we turned our attention to reads that map to the C-terminal region coded by exons 3-12 of MUC3A and $M U C 3 B$, where we identified in average $3.5 \pm 1.6$ unique reads per kilobase transcript (RPK) of MUC3A and 13.0 \pm 4.4 unique RPK of MUC3B (Figure 5A, Supplementary table 3). We next used unique and shared reads in the $C$-terminal region to calculate normalized gene expression of MUC3A, MUC3B, MUC12 and MUC17 in human intestine. In ileum, MUC17 showed significantly higher expression than MUC3A, MUC3B and MUC12, while MUC12 expression showed a trend towards higher expression in rectum compared to ileum. We detected comparable numbers of $M U C 3 A$ and $M U C 3 B$ transcripts in all three intestinal segments (Figure 5B). We next applied a targeted reverse transcriptase quantitative polymerase chain reaction (RT-qPCR) to validate the presence of unique MUC3A and MUC3B transcripts in ileum collected from five human patients (Supplementary table 4). For this purpose, we designed gene-specific primer pairs targeting sequences in exons 3 and 8 with 9.5-12.0\% mismatch between MUC3A and MUC3B. The resulting 645 bp cDNA amplicons from each transcript were further distinguishable by a unique Pstl restriction site in MUC3A cDNA amplicon (Figure 5C). RT-qPCR from all five patients resulted in the expected $646 \mathrm{bp}$ cDNA amplicon and subsequent Pst/-digestion produced 380 bp and 266 bp restriction fragments (Figure 5C). Notably, we observed significant differences in intensities of Pst/sensitive and $P$ st/-resistant fragments produced by the two gene-specific primer pairs. $75 \%$ of amplicons generated by MUC3A-specific primers were Pst/-sensitive and therefore originated from MUC3A transcripts (Figure 5D). Similarly, $76 \%$ of amplicons generated by MUC3Bspecific primers were Pst/-resistant MUC3B transcripts. Thus, despite significant sequence similarity between the two MUC3 genes, we successfully identified and distinguished between MUC $3 A$ and MUC3B transcripts in the human intestine.

\section{Completion of a gapless human MUC3 cluster at locus $7 q 22$}

Finally, based on the T2T-CHM13 assembly, we revised the gapless length of all four membrane mucins genes in the MUC3 cluster at locus 7q22. In this new assembly, we identified a longer PTS-encoding exon 2 of 15,870 nt in MUC3A compared to $8805 \mathrm{nt}$ in GRCh38.p13. The PTS-encoding exon of human MUC12 was 32,428 nt long compared to 14,935 nt in GRCh38.p13 (Supplementary table 5). The complete gapless sequences of MUC3A, MUC3B, MUC12 and MUC17 genes at 7q22 locus are publicly available via Mucin database X.X 2021 (http://www.medkem.gu.se/mucinbiology/databases/index.html). 


\section{Discussion}

Mucin genes containing long protein-coding sequences consisting of tandem repeats that are difficult to read and measure. As results, many human mucin gene sequences remain uncomplete. Sequence gaps also appear in the genome of Mus musculus, an important model organism for understanding human gene function. In an attempt to fill these knowledge gaps in mucin genetics, we focused on a cluster of three membrane mucins genes, the MUC3 cluster, at locus q22 in human chromosome 7. The MUC3 cluster is conserved in the cercopithecoid and hominoid superfamilies, where two distinct MUC $3 A$ and MUC3B genes are annotated in all species except in $H$. sapiens and G. gorilla. In this study, we leveraged the recent T2T-CHM13 assembly to fill a $60 \mathrm{~kb}$ sequence gap sandwiched between MUC3A and MUC12 genes. Sequence alignments revealed that this membrane mucin gene shares high structural and sequence similarity with MUC3A; it consists of a total 12 exons and carries a PTS-encoding exon 2 encompassing imperfect and perfect tandem repeats that were conserved in MUC3A. Moreover, within the gap we identified a SEA domain, a transmembrane domain and a cytoplasmic tail with a Class I PDZ motif that is conserved in all annotated membrane mucins of the MUC3 cluster. Importantly, nucleotide mismatches in introns and exons clearly distinguished this putative MUC3B gene from MUC3A. Also, lengths of intergenic regions spanning across $M U C 3 A$, the putative $M U C 3 B$ and MUC12 corresponded to intergenic lengths observed within the MUC3 cluster of cercopithecoids and hominoids.

The evolutionary conservation of $M U C 3 A$ and $M U C 3 B$ genes further suggests that their regulation is conserved in higher mammals. Comparative sequence alignments and available DNase I- and ChIP-seq data sets uncovered a conserved cis-regulatory element upstream of MUC3B that included binding sites for transcription factors HNF4A, HNF4G and STAT3. Notably, HNF4A and HNF4G regulate expression of genes that encode critical brush border proteins in the apical membrane domain of transporting intestinal epithelial cells (IECs) (Chen et al., 2021). STAT3 acts downstream of the heteromeric epithelial cell receptor for the cytokine IL-22 that regulates expression of MUC17, which builds the protective glycocalyx barrier atop transporting IECs (Layunta et al., 2021). Finally, mapping published RNA-seq data sets to the T2T-CHM13 assembly identified unique sequencing reads for MUC3A and MUC3B genes in the human intestine, while gene expression was absent in liver and kidneys. We also validated high-through put expression data by a targeted quantitative detection of distinct MUC3A and MUC3B transcripts in the human ileum. Collectively, we identified a previously unannotated $M U C 3 B$ gene at locus $7 q 22$ and provide evidence for its expression in human intestinal epithelial cells.

The MUC3B gene emerges first in the parvorder Catarrhini (Old World monkeys) but is absent in Platyrrhini (New World monkeys), suggesting a gene duplication event in the Simian 
infraorder 43 Mya. Interestingly, N- and C-terminal regions of MUC3A and MUC3B are highly conserved within Catarrhini, whereas the PTS-encoding exons exhibit higher evolutionary divergence. The PTS domains of membrane mucins genes are encoded by short nucleotide sequences organized in tandem repeats. PTS domains are generally poorly conserved (Lang et al., 2007), as individual repeats are added or removed through recombination to generate variable number tandem repeats (VNTRs) that introduce substantial gene polymorphism. In analogy with other genes carrying VNTRs (Sulovari et al., 2019), our study shows that tandem repeat regions of MUC3 cluster genes have undergone expansion during primate evolution. Low conservation and considerable polymorphism between and within species suggest that O-glycosylation of mucin VNTRs is a non-template-driven process that occurs in a specific evolutionary and environmental context with the primary aim of attaching specific glycan epitopes to the mucin protein backbone. Therefore, glycosylation mucin VNTRs in microberich environments such as the oral cavity and gastrointestinal tract are likely under selective pressure to maintain appropriate interactions with microorganisms that have coevolved with the host through various periods of geographical, dietary and life-style adaptations. This cospeciation is exemplified within the human gut microbiome, where the microbiome of presentday humans is enriched in mucin-degrading genes compared to enrichment of starch- and chitin-degrading genes in our ancestral gut microbiome (Wibowo et al., 2021). Another example is found at the epithelial surface glycocalyx, which underwent major remodeling $>2$ Mya when a human ancestor acquired an inactivating mutation in $\mathrm{CMAH}$, a gene responsible for converting $\mathrm{N}$-acetylneuraminic acid (Neu5Ac) to $\mathrm{N}$-glycolylneuraminic acid (Neu5Gc) (Chou et al., 2002). The resulting accumulation of terminal Neu5Ac in the glycocalyx of human cells has since be exploited by numerous pathogenic microorganism such as Vibrio cholera (Alisson-Silva et al., 2018).

Due to existing challenges in sequencing very long repetitive regions, the nature of mucin polymorphism and its contribution to human disease phenotypes remains elusive. A recent study showed that the length of VNTRs in membrane mucin MUC1 is associated with several phenotypes related to kidney function (Mukamel et al., 2021), supporting the notion that glycosylated PTS domains of membrane mucins play critical roles in organ function and homeostasis. Intestinal membrane mucin MUC17 is genetically and structurally related with MUC3A and MUC3B and functions as a major building block of the dense glycocalyx covering transporting IECs. In mouse small intestine, Muc17 expression is induced during the sucklingweaning transition when density and complexity of the gut microbiota increases and IECs establish a cell-attached glycocalyx to prevent adhesion of luminal bacteria to the epithelium (Layunta et al., 2021). While the function of the MUC3A, MUC3B and MUC12 remains elusive, their expression varies along different segments of the human intestine, suggesting that MUC3 
cluster genes perform segment- and cell-specific functions in humans and other mammalian vertebrates. Our comprehensive map of the MUC3 cluster in the human genome provides opportunities to identify new VNTR polymorphisms associated with disease phenotypes and allow for future exploration of experimental mammalian models such as the mouse for gene orthologs of the MUC3 cluster.

\section{Methods}

\section{Recruitment of patients and sample collection}

Patients $\geq 18$ years who were referred to Sahlgrenska University Hospital (Gothenburg, Sweden) for colonoscopy, were eligible for inclusion and subject to the provision of written informed consent. Patients with macroscopic/microscopic evidence of ileocolonic pathology other than inflammatory bowel disease were excluded. Eight biopsies were obtained from the terminal ileum of each patient. The study protocol was approved by the regional ethics committee (Ethical permit \#2020-03196) and was in compliance with the Declaration of Helsinki.

\section{Phylogenic data}

Phylogenic trees and molecular time estimates were extracted from TimeTree (Hedges et al., 2006, 2015).

\section{Sequence alignments}

Local sequence similarity search and identity measurements of MUC genes was performed using NCBI BLAST (McGinnis and Madden, 2004). Multiple sequence alignment of MUC gene and protein homologues were conducted using CLUSTALW (Larkin et al., 2007). Perl scripts were used for all data extraction (see supplementary information). Promotor regions $-1 \mathrm{~kb}$ from transcription start site of $M U C 3 A$ and $M U C 3 B$ in cercopithecoid and hominoid superfamilies were aligned using Multiple Alignment using Fast Fourier Transform (MAFFT) high speed multiple sequence alignment tool (Madeira et al., 2019).

\section{Generation of dotplots for pairwise sequence alignment and sequence logo representations}

Dotplots representing pairwise sequence alignments were generated using Genome Pair Rapid Dotter (GEPARD) version 1.40 (Krumsiek et al., 2007). Sequence logos of perfect tandem repeats were generated using WebLogo3 (Crooks et al., 2004).

\section{Mapping of DNase-seq and ChIP-seq data to the human genome}

DNase hypersensitive sequences upstream of MUC3A in GRCh38 and Chromatin immunoprecipitation (ChIP) sequencing of human small intestine, colon and stomach samples 
are summarized in Supplementary table 6 (Zhang et al., 2020). Graphical representation of epigenic signatures was prepared by aggregating multiple segment-sorted tracks using the Matplot function in Washington University Epigenome Browser v53.5.0 (Li et al., 2019).

\section{Single-cell expression of transcription factors}

Expression profiles for transcription factors were extracted from the following data sets available at Single Cell Portal (Broad institute): single-cell transcriptome analysis of human small intestine (GSE148829) (Ziegler et al., 2020), human colon (GSE178341) (Pelka et al., 2021) and mouse small intestine (GSE92332) (Haber et al., 2017).

\section{Mapping of RNA-sequencing data to T2T-CHM13 human genome assembly}

The T2T-CHM13 human genome assembly was downloaded from NCBI BioProject PRJNA559484). Fastq-dump was used to obtain RNA-sequencing reads. Burrows-Wheeler Aligner (BWA) software package ( $\mathrm{Li}$ and Durbin, 2009) was used to align RNA-sequencing reads to exonic sequences of genes belonging to the MUC3 cluster. Perl scripts were used to perform quality control and measure read number (see supplementary methods). The following publicly available data sets were used to determine MUC gene expression in human tissues: single-cell transcriptome analysis of human ileum, colon, rectum (GSE125970) (Wang et al., 2020), human liver (GSE124395) (Aizarani et al., 2019) and human kidney (GSE131685) (Aizarani et al., 2019). Gene expression of individual MUC genes in the MUC3 cluster was calculated as transcripts per million (TPM) as previously described (Li et al., 2010).

\section{RNA extraction from human ileum, cDNA synthesis and RT-qPCR}

RNA from human ileal biopsies was extracted using RNeasy Mini Kit (Qiagen). 500 ng of RNA was reverse transcribed to cDNA using TaqMan Reverse Transcription kit (\#N8080234, Applied Biosystems) using $2.5 \mu \mathrm{M}$ random primers using the cycling parameters $25.0^{\circ} \mathrm{C}$ for $10 \mathrm{~min}, 37.0^{\circ} \mathrm{C}$ for $30 \mathrm{~min}$, and $95.0^{\circ} \mathrm{C}$ for $5 \mathrm{~min}$. $750 \mathrm{ng}$ of cDNA was used for downstream reverse transcription quantitative PCR (RT-qPCR) with $0.3 \mu \mathrm{M}$ MUC3A-specific primers (forward 5'-TGGGGGTCAGTGGGATGGCCTCAAA-3'; 5'CACGTGGGACCGCTCGTCTCC) and MUC3B-specific primers (forward 5'CGGGGGCCAGTGGGATGGCCTCAAG-3'; reverse 5'-CACGCGGGACCGCTCGTCTCT-3') using SsoFast EvaGreen Supermix (\#1725200, Bio-Rad) on a CFX96 Real-Time PCR Detection System (Bio-Rad) with the cycling parameters $95.0^{\circ} \mathrm{C}$ for $3 \mathrm{~min}, 39$ cycles of $95.0^{\circ} \mathrm{C}$ for $10 \mathrm{~s}, 63.5^{\circ} \mathrm{C}$ for $10 \mathrm{~s}, 72.0^{\circ} \mathrm{C}$ for $20 \mathrm{~s}$. Melting curve analysis was performed at $95.0^{\circ} \mathrm{C} 10$ $\mathrm{s}$ and $65.0^{\circ}$ to $95.0^{\circ} \mathrm{C}$ at an increment of $5^{\circ} \mathrm{C}$ for $5 \mathrm{~s}$.

\section{Restriction site analysis and agarose gel electrophoresis}


$5 \mu \mathrm{L}$ of RT-qPCR reaction was digested with $1 \mu \mathrm{L}$ FastDigest Pst/ restriction enzyme (\#FD0614, ThermoFisher Scientific) for $1 \mathrm{~h}$ at $37^{\circ} \mathrm{C}$. Full-length amplicons and digestion products were separated on $1.5 \%$ agarose gel with ethidium bromide.

\section{Statistics}

Statistical analysis and graphical illustrations were performed using GraphPad PRISM 8.3.1 (GraphPad Software). Data are presented as mean \pm standard deviation (SD). Statistical tests were applied as indicated. For all statistical analyses: ${ }^{*} p<0.05,{ }^{* *} p<0.01,{ }^{* * *} p<0.0001,{ }^{* * * *}$ $\mathrm{p}<0.00001, \mathrm{~ns}=$ non-significant.

\section{Author contributions}

T.L. collected the data, contributed data and performed analysis. T.P. conceived and designed the analysis, collected data, contributed data, performed analysis and wrote the manuscript.

\section{Acknowledgements}

We thank Professor Gunnar C. Hansson for valuable discussions. This work was supported by the Swedish Society for Medical Research (Svenska Sällskapet för Medicinsk Forskning, grant S17-0005), National Institutes of Health (grants 5U01AI095542-08-WU-19-95 and 5U01AI095542-09-WU-20-77), Wenner-Gren Foundations (grants FT2017-0002, UPD20180065, and WUP2017-0005), Jeansson Foundations (grant JS2017-0003), and the Åke Wiberg Foundation (grant M17-0062).

\section{References}

Aizarani, N., Saviano, A., Sagar, Mailly, L., Durand, S., Herman, J.S., Pessaux, P., Baumert, T.F., and Grün, D. (2019). A Human Liver Cell Atlas reveals Heterogeneity and EpithelialProgenitors. Nature 572, 199.

Alisson-Silva, F., Liu, J.Z., Diaz, S.L., Deng, L., Gareau, M.G., Marchelletta, R., Chen, X., Nizet, V., Varki, N., Barrett, K.E., et al. (2018). Human evolutionary loss of epithelial Neu5Gc expression and species-specific susceptibility to cholera. PLoS Pathog. 14.

Chaisson, M.J.P., Wilson, R.K., and Eichler, E.E. (2015). Genetic variation and the de novo assembly of human genomes. Nat. Rev. Genet. 16, 627.

Chen, L., Luo, S., Dupre, A., Vasoya, R.P., Parthasarathy, A., Aita, R., Malhotra, R., Hur, J., Toke, N.H., Chiles, E., et al. (2021). The nuclear receptor HNF4 drives a brush border gene program conserved across murine intestine, kidney, and embryonic yolk sac. Nat. Commun. 2021121 12, 1-15.

Chou, H.H., Hayakawa, T., Diaz, S., Krings, M., Indriati, E., Leakey, M., Paabo, S., Satta, Y., Takahata, N., and Varki, A. (2002). Inactivation of CMP-N-acetylneuraminic acid 
hydroxylase occurred prior to brain expansion during human evolution. Proc. Natl. Acad. Sci. 99, 11736-11741.

Craig Venter, J., Adams, M.D., Myers, E.W., Li, P.W., Mural, R.J., Sutton, G.G., Smith, H.O., Yandell, M., Evans, C.A., Holt, R.A., et al. (2001). The sequence of the human genome.

Science (80-. ). 291, 1304-1351.

Crooks, G.E., Hon, G., Chandonia, J.M., and Brenner, S.E. (2004). WebLogo: A Sequence Logo Generator. Genome Res. 14, 1188.

Fornes, O., Castro-Mondragon, J.A., Khan, A., Van Der Lee, R., Zhang, X., Richmond, P.A., Modi, B.P., Correard, S., Gheorghe, M., Baranašić, D., et al. (2020). JASPAR 2020: update of the open-access database of transcription factor binding profiles. Nucleic Acids Res. 48, D87.

Haber, A.L., Biton, M., Rogel, N., Herbst, R.H., Shekhar, K., Smillie, C., Burgin, G., Delorey, T.M., Howitt, M.R., Katz, Y., et al. (2017). A single-cell survey of the small intestinal epithelium. Nature 551, 333-339.

Hedges, S.B., Dudley, J., and Kumar, S. (2006). TimeTree: a public knowledge-base of divergence times among organisms. Bioinformatics 22, 2971-2972.

Hedges, S.B., Marin, J., Suleski, M., Paymer, M., and Kumar, S. (2015). Tree of Life Reveals Clock-Like Speciation and Diversification. Mol. Biol. Evol. 32, 835.

Krumsiek, J., Arnold, R., and Rattei, T. (2007). Gepard: a rapid and sensitive tool for creating dotplots on genome scale. Bioinformatics 23, 1026-1028.

Lander, E.S., Linton, L.M., Birren, B., Nusbaum, C., Zody, M.C., Baldwin, J., Devon, K., Dewar, K., Doyle, M., Fitzhugh, W., et al. (2001). Initial sequencing and analysis of the human genome. Nat. 20014096822 409, 860-921.

Lang, T., Hansson, G.C., and Samuelsson, T. (2007). Gel-forming mucins appeared early in metazoan evolution. Proc Natl Acad Sci U S A 104, 16209-16214.

Lang, T., Klasson, S., Larsson, E., Johansson, M.E.V., Hansson, G.C., and Samuelsson, T. (2016). Searching the Evolutionary Origin of Epithelial Mucus Protein Components-Mucins and FCGBP. Mol. Biol. Evol. 33, 1921.

Larkin, M.A., Blackshields, G., Brown, N.P., Chenna, R., Mcgettigan, P.A., McWilliam, H., Valentin, F., Wallace, I.M., Wilm, A., Lopez, R., et al. (2007). Clustal W and Clustal X version 2.0. Bioinformatics 23, 2947-2948.

Layunta, E., Jäverfelt, S., Dolan, B., Arike, L., and Pelaseyed, T. (2021). IL-22 promotes the formation of a MUC17 glycocalyx barrier in the postnatal small intestine during weaning. Cell 
Rep. 34, 108757.

Li, H., and Durbin, R. (2009). Fast and accurate short read alignment with Burrows-Wheeler transform. Bioinformatics 25, 1754.

Li, B., Ruotti, V., Stewart, R.M., Thomson, J.A., and Dewey, C.N. (2010). RNA-Seq gene expression estimation with read mapping uncertainty. Bioinformatics 26, 493-500.

Li, D., Hsu, S., Purushotham, D., Sears, R.L., and Wang, T. (2019). WashU Epigenome Browser update 2019. Nucleic Acids Res. 47, W158-W165.

Ligtenberg, M.J.L., Kruijshaar, L., Buijs, F., Van Meijer, M., Litvinov, S. V., and Hilkens, J. (1992). Cell-associated episialin is a complex containing two proteins derived from a common precursor. J Biol Chem 267, 6171-6177.

Madeira, F., Park, Y.M., Lee, J., Buso, N., Gur, T., Madhusoodanan, N., Basutkar, P., Tivey, A.R.N., Potter, S.C., Finn, R.D., et al. (2019). The EMBL-EBI search and sequence analysis tools APIs in 2019. Nucleic Acids Res. 47, W636-W641.

Malmberg, E.K., Pelaseyed, T., Petersson, Å.C., Seidler, U.E., De Jonge, H., Riordan, J.R., and Hansson, G.C. (2008). The C-terminus of the transmembrane mucin MUC17 binds to the scaffold protein PDZK1 that stably localizes it to the enterocyte apical membrane in the small intestine. Biochem. J. 410, 283-289.

McGinnis, S., and Madden, T.L. (2004). BLAST: at the core of a powerful and diverse set of sequence analysis tools. Nucleic Acids Res. 32, W20.

Mukamel, R.E., Handsaker, R.E., Sherman, M.A., Barton, A.R., Zheng, Y., McCarroll, S.A., and Loh, P.R. (2021). Protein-coding repeat polymorphisms strongly shape diverse human phenotypes. Science (80-. ). 373, 1499-1505.

Nason, R., Büll, C., Konstantinidi, A., Sun, L., Ye, Z., Halim, A., Du, W., Sørensen, D.M., Durbesson, F., Furukawa, S., et al. (2021). Display of the human mucinome with defined Oglycans by gene engineered cells. Nat. Commun. 2021121 12, 1-16.

Nurk, S., Koren, S., Rhie, A., Rautiainen, M., Bzikadze, A. V., Mikheenko, A., Vollger, M.R., Altemose, N., Uralsky, L., Gershman, A., et al. (2021). The complete sequence of a human genome. BioRxiv 2021.05.26.445798.

Pei, J., and Grishin, N. V. (2017). Expansion of divergent SEA domains in cell surface proteins and nucleoporin 54. Protein Sci. 26, 617.

Pelaseyed, T., and Hansson, G.C. (2020). Membrane mucins of the intestine at a glance. J. Cell Sci. 133, jcs240929.

Pelaseyed, T., Zäch, M., Petersson, Å.C., Svensson, F., Johansson, D.G.A.A., and 
Hansson, G.C. (2013). Unfolding dynamics of the mucin SEA domain probed by force spectroscopy suggest that it acts as a cell-protective device. FEBS J. 280, 1491-1501.

Pelka, K., Hofree, M., Chen, J.H., Sarkizova, S., Pirl, J.D., Jorgji, V., Bejnood, A., Dionne, D., Ge, W.H., Xu, K.H., et al. (2021). Spatially organized multicellular immune hubs in human colorectal cancer. Cell 184, 4734-4752.e20.

Pratt, W.S., Crawley, S., Hicks, J., Ho, J., Nash, M., Kim, Y.S., Gum, J.R., and Swallow, D.M. (2000). Multiple Transcripts of MUC3: Evidence for Two Genes, MUC3A and MUC3B. Biochem Biophys Res Commun 275, 916-923.

Sandelin, A., Alkema, W., Engström, P., Wasserman, W.W., and Lenhard, B. (2004). JASPAR: an open-access database for eukaryotic transcription factor binding profiles. Nucleic Acids Res. 32, D91.

Shi, L., Guo, Y., Dong, C., Huddleston, J., Yang, H., Han, X., Fu, A., Li, Q., Li, N., Gong, S., et al. (2016). Long-read sequencing and de novo assembly of a Chinese genome. Nat. Commun. 201671 7, 1-10.

Su, C., Lv, Y., Lu, W., Yu, Z., Ye, Y., Guo, B., Liu, D., Yan, H., Li, T., Zhang, Q., et al. (2021). Single-Cell RNA Sequencing in Multiple Pathologic Types of Renal Cell Carcinoma Revealed Novel Potential Tumor-Specific Markers. Front. Oncol. 11, 719564.

Sulovari, A., Li, R., Audano, P.A., Porubsky, D., Vollger, M.R., Logsdon, G.A., Warren, W.C., Pollen, A.A., Chaisson, M.J.P., and Eichler, E.E. (2019). Human-specific tandem repeat expansion and differential gene expression during primate evolution. Proc. Natl. Acad. Sci. U. S. A. 116, 23243-23253.

Svensson, F., Lang, T., Johansson, M.E.V., and Hansson, G.C. (2018). The central exons of the human MUC2 and MUC6 mucins are highly repetitive and variable in sequence between individuals. Sci. Reports 201881 8, 1-10.

Wang, Y., Song, W., Wang, J., Wang, T., Xiong, X., Qi, Z., Fu, W., Yang, X., and Chen, Y.G. (2020). Single-cell transcriptome analysis reveals differential nutrient absorption functions in human intestine. J. Exp. Med. 217.

Wibowo, M.C., Yang, Z., Borry, M., Hübner, A., Huang, K.D., Tierney, B.T., Zimmerman, S., Barajas-Olmos, F., Contreras-Cubas, C., García-Ortiz, H., et al. (2021). Reconstruction of ancient microbial genomes from the human gut. Nat. 20215947862 594, 234-239.

Zhang, J., Lee, D., Dhiman, V., Jiang, P., Xu, J., McGillivray, P., Yang, H., Liu, J., Meyerson, W., Clarke, D., et al. (2020). An integrative ENCODE resource for cancer genomics. Nat. Commun. $202011111,1-11$. 
Ziegler, C.G.K., Allon, S.J., Nyquist, S.K., Mbano, I.M., Miao, V.N., Tzouanas, C.N., Cao, Y., Yousif, A.S., Bals, J., Hauser, B.M., et al. (2020). SARS-CoV-2 Receptor ACE2 Is an Interferon-Stimulated Gene in Human Airway Epithelial Cells and Is Detected in Specific Cell Subsets across Tissues. Cell 181, 1016.

\section{Figure legends}

Figure 1. Conservation of MUC3 cluster in Cercopithecoid and Hominoid superfamilies. A) MUC3 cluster in the current GRCh38.p12 assembly is flanked by genes ACHE, TRIM56 and SERPINE1 at locus q22 in human chromosome 7.

B) Members of the cercopithecoid and hominoid superfamilies, with the exception of $H$. sapiens and G. gorilla, carry a MUC3 cluster consisting of MUC3A, MUC3B, MUC12 and MUC17 genes.

C) Emergence of MUC3B gene in MUC3 cluster in Catarrhini parvorder (filled black circles). Open circles indicated presence of MUC3, MUC12 and MUC17 genes in Platyrrhini parvorder. NA indicates lack of sufficient sequence information for detection of MUC3 cluster.

Figure 2. Evidence of a putative MUC3B gene in recent human genome assemblies.

A) Exploration of PacBio sequencing of HX1 genome identified SMRT reads covering the $\mathrm{N}$ intergenic sequences between MUC3A and putative MUC3B, an incomplete PTS sequence and intergenic sequences between putative MUC3B and MUC12.

B) The T22-CHM13 assembly contains a $60 \mathrm{~kb}$ gap between MUC3A and MUC12.

C) Sequence alignment of SEA, transmembrane (TM) and cytoplasmic tails (CT) of MUC3A and putative $M U C 3 B$ shows high sequence identity, nucleotide mismatches and a conserved PDZ binding motif.

Figure 3. Comparison of genetic and structural features of MUC3A and MUC3B genes.

A) Exon structure and length of exon 2 of MUC3A and MUC3B.

B) Evolutionary rate of $\mathrm{N}$-terminal-, PTS- and C-terminal-encoding exons in $M U C 3 A, M U C 3 B$, MUC12 and MUC17 measured as gene content conservation (\%) versus evolutionary distance (Mya).

C) Dotplot of pairwise sequence alignment of $M U C 3 A$ and $M U C 3 B$ identified imperfect (blue) and perfect (red) tandem repeat sequences in exon 2 .

D) Sequence logo representation of 166 and 549 perfect tandem repeats (TRs) in exon 2 of $M U C 3 A$ and $M U C 3 B$, respectively.

Figure 4. Conserved regulatory elements upstream of $M U C 3 A$ and $M U C 3 B$ genes. 
A) Epigenetic analysis of human small intestine and colon reveals a DNase I-sensitive candidate cis-regulatory element (cCRE) and specific histone modifications surrounding MUC3A transcription start site.

B) Single cell analysis of human and mouse intestine shows gene expression of transcription factors in transporting intestinal epithelial cells (enterocytes and colonocytes) with conserved binding sites upstream of MUC3A and MUC3B.

C) Binding sites for transcription factors STAT3 and HNF4A/G are completely conserved upstream of $M U C 3 A$ and $M U C 3 B$ in cercopithecoid and hominoid superfamilies.

\section{Figure 5. Expression of MUC3B gene in human intestine.}

A) Unique reads for $M U C 3 A$ and $M U C 3 B$ in RNA-sequencing data from human ileum, colon and rectum mapped to T2T-CHM13 human genome assembly.

B) Gene expression of MUC3 cluster genes in human ileum, colon and rectum. 2 samples per tissue segments. * $p<0.05$ as determined by two-way ANOVA, corrected for multiple comparison using Tukey's test. Data are presented as mean \pm standard deviation (SD).

C) Specific primers amplify 646 bp cDNAs spanning exons 3-8 in MUC3A and MUC3B transcripts from ileum of five individuals. MUC3A cDNA carries a Pst/ restriction site in exon 6 that distinguishes MUC3A from MUC3B transcripts. Agarose gel electrophoresis of PstI restriction digests of amplified cDNA from $M U C 3 A$ and $M U C 3 B$ transcripts results in $380 \mathrm{bp}$ and 266 bp fragments from MUC3A cDNA.

D) Quantification of bands from agarose gel in C. $n=5$ individuals. Data are presented as mean \pm standard deviation (SD). 
Fiqupe®Pxiv preprint doi: https://doi.org/10.1101/2021.12.31.474548; this version posted January 1, 2022. The copyright holder for this preprint Furich was not certified by peer review) is the author/funder, who has granted bioRxiv a license to display the preprint in perpetuity. It is A $7 q 22$

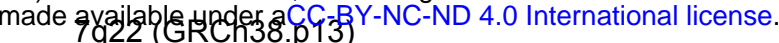

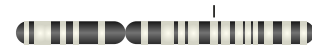

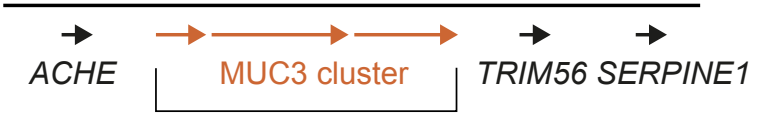

B
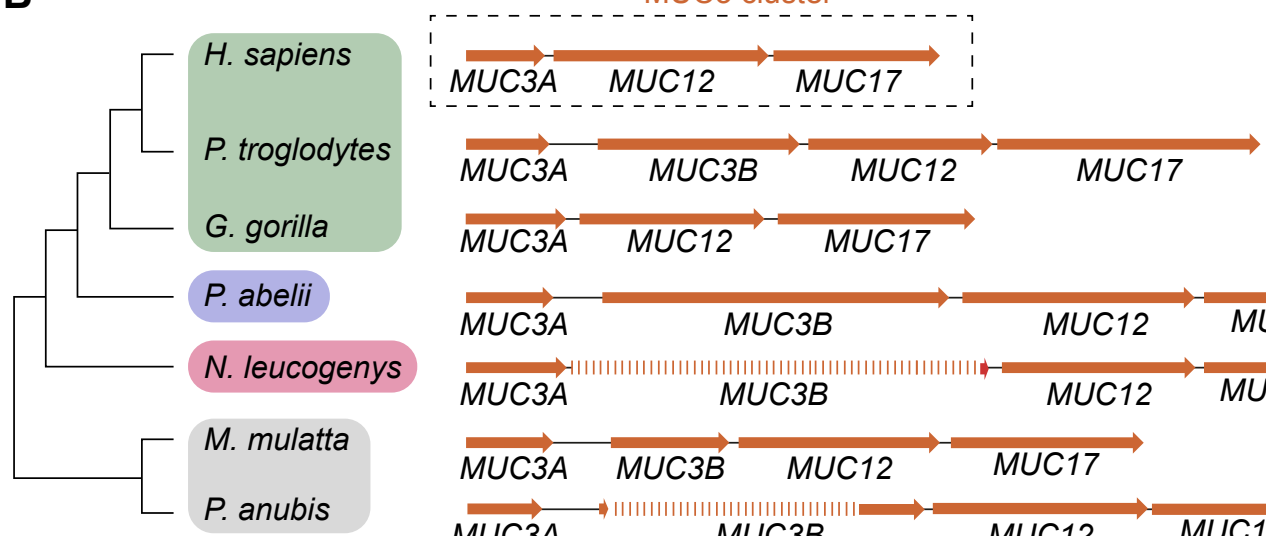

$\overrightarrow{M U C 3 A} \mathrm{MUC3B} \rightarrow \overrightarrow{M U C 12} \rightarrow \overrightarrow{M U C 17}$

$\overrightarrow{M U C 3 A} M U C 3 B \rightarrow$

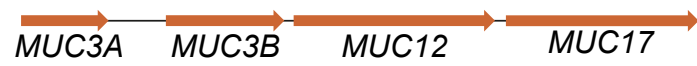

\begin{tabular}{cccccc} 
MUC3A & \multicolumn{3}{c}{ MUC3B } & MUC12 & \multicolumn{2}{c}{ MUC17 } \\
0 & 50 & 100 & 150 &
\end{tabular}

Cercopithecinae

Nomascus

Ponginae

Homininae

C

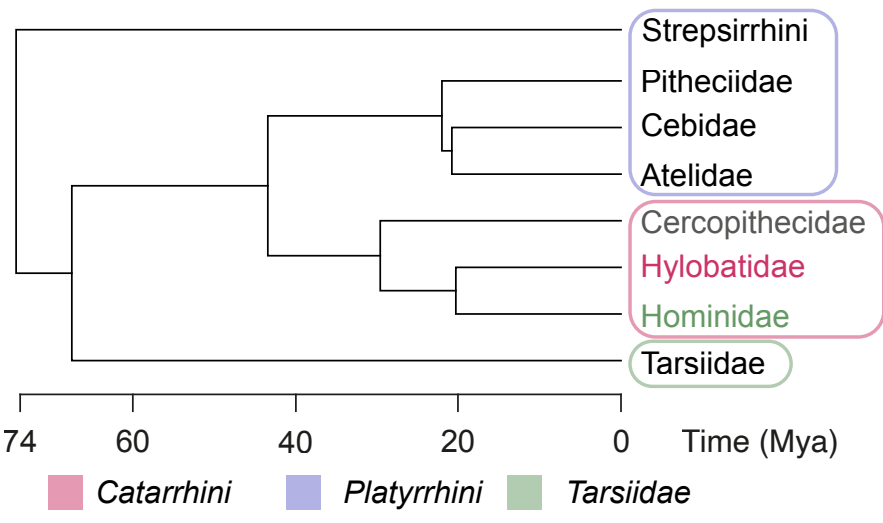

\begin{tabular}{|c|c|c|c|}
\hline $3 A$ & $3 B$ & 12 & 17 \\
\hline$\bigcirc$ & & $\bigcirc$ & $\bigcirc$ \\
\hline NA & NA & NA & NA \\
\hline$\bigcirc$ & & $\bigcirc$ & $\bigcirc$ \\
\hline NA & NA & NA & NA \\
\hline$\bigcirc$ & $\bigcirc$ & $\bigcirc$ & $\bigcirc$ \\
\hline$\bigcirc$ & $\bigcirc$ & $\bigcirc$ & $\bigcirc$ \\
\hline$\bigcirc$ & $\bigcirc$ & $\bigcirc$ & $\bigcirc$ \\
\hline NA & NA & NA & NA \\
\hline
\end{tabular}
Catarrhini
Platyrrhini
Tarsiidae 
Fiqupiomiv preprint doi: https://doi.org/10.1101/2021.12.31.474548; this version posted January 1, 2022. The copyright holder for this preprint fich was not certified by peer review) is the author/funder, who has granted bioRxiv a license to display the preprint in perpetuity. It is

A GRCh38.p13

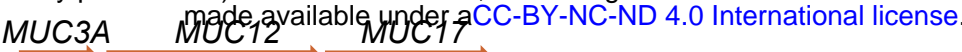

SMRT reads

\begin{tabular}{r|cc}
\hline 6,187 & 10,431 & 2,217 \\
\hline 5,123 & 12,695 & 2,247 \\
886 & 9,692 & 2,017
\end{tabular}

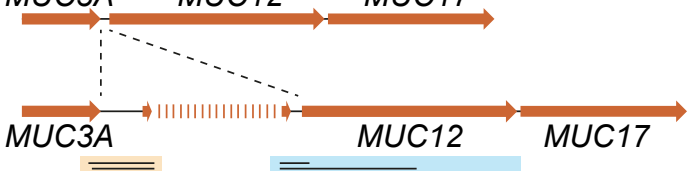

$-\cdot-\cdot-\cdot-$

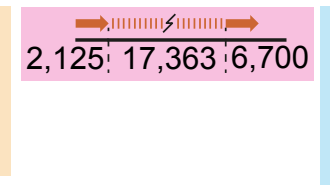

\begin{tabular}{l:l:l}
\hline 5,675 & 2,338 & 6,293 \\
\hline 6,081 & 2,560 & 16,654 \\
\hline 5,771 & 2,412 & 8,385 \\
\hline 6,265 & 2,526 & 7,158 \\
\hline 6,335 & 2,511 & 13,805
\end{tabular}

B

GRCh38.p13

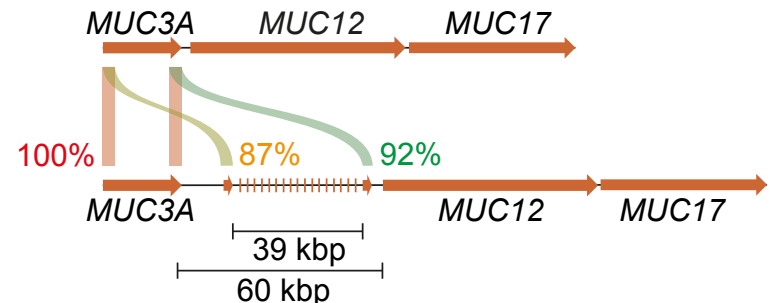

C

T2T-CHM13

$60 \mathrm{kbp}$

SEA

TM CT

\begin{abstract}
MUC3A SEA MUC3B_SEA

$\begin{array}{llllllllllllllllllll}\mathrm{D} & \mathrm{V} & \mathrm{V} & \mathrm{E} & \mathrm{T} & \mathrm{E} & \mathrm{V} & \mathrm{G} & \mathrm{M} & \mathrm{E} & \mathrm{V} & \mathrm{S} & \mathrm{V} & \mathrm{D} & \mathrm{Q} & \mathrm{Q} & \mathrm{F} & \mathrm{S} & \mathrm{P} & \mathrm{D}\end{array}$ GATGTAGTGGAGACCGAGGTGGGCATGGAAGTGTCTGTGGATCAGCAGTTCTCGCCGGAC 60

MUC3A SEA $\begin{array}{llllllllllllllllllll}\text { L } & N & D & N & T & S & O & A & Y & R & D & F & N & K & T & F & W & N & Q & M\end{array}$ MUC3B_SEA CTCAATGACAACACTTCCCAGGCCTACAGGGATTTCAACAAGACCTTCTGGAATCAGATG 120 CTCAATGACAACACTTCCCAGGCCTACAGGGATTTCAACAAGACCTTCTGGAATCAGATG 120

MUC3A SEA MUC 3B SEA $\begin{array}{lllllllllllllllllllllll}\mathrm{Q} & \mathrm{K} & \mathrm{I} & \mathrm{F} & \mathrm{A} & \mathrm{D} & \mathrm{M} & \mathrm{Q} & \mathrm{G} & \mathrm{F} & \mathrm{T} & \mathrm{F} & \mathrm{K} & \mathrm{G} & \mathrm{V} & \mathrm{E} & \mathrm{I} & \mathrm{L} & \mathrm{S} & \mathrm{L} & \\ \text { CAGAAAGATTTTTGCAGACATGCAGGGCTTCACCTTCAAGGGTGTTGGAGATCCTGTCCCTG } & 180\end{array}$ CAGAAGATTTTTGCAGACATGCAGGGCTTCACCTTCAAGGGTGTGGAGATCCTGTCCCTG 180 $\begin{array}{lllllllllllllllllllll}R & N & G & S & I & V & V & D & Y & \text { L } & \text { V } & \text { L } & \text { L } & \text { E } & \text { M } & \text { P } & \text { F } & \text { S } & \text { P } & Q\end{array}$ MUC3A_SEA AGGAATGGCAGCATCGTGGTGGACTACCTGGTCCTGCTGGAGATGCCCTTCAGCCCCCAG 240 MUC3B_SEA AGGAATGGCAGCATCGTGGTGGACTACCTGGTCCTGCTGGAGATGCCCTTCAGTCCCCAG 240

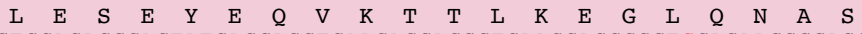

MUC3A SEA MUC 3B-SEA CTATGAGCAGGTGAAGACCACGCTGAAGGAGGGGCTGCAGAACGCCAGC 300

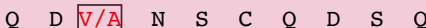

MUC3A SEA CAGGATGTGAACAGCTGCCAGGACTCCCAG 330

MUC3B_SEA CAGGATGCGAACAGCTGCCAGGACTCCCAG 330

$\begin{array}{lllllllllllllllllllllll} & \text { V } & \text { G } & \text { G } & \text { L } & \text { T } & \text { A } & \text { G } & \text { A } & \text { A } & \text { L } & \text { L } & \text { V } & \text { L } & \text { L } & \text { L } & \text { L } & \text { A } & \text { L } & \text { G } & \text { V } & & \end{array}$ MUC3A_TM GTCGGGGGCCTGACGGCCGGCGCCGCGCTGCTGGTGCTGCTGCTGCTGGCGCTGGGCGTC 60 MUC3B_TM GTCGGGGGCCTGACGGCCGGCGCCGCGTGCTGGTGCTGCTGCTGCTGGCGCTGGGCGTC 60

$\begin{array}{llllllllllllllllllllll} & & R & A & V & R & S & G & W & W & G & G & Q & R & R & G & R & S & W & D & Q & D\end{array}$ CGGGCGGTGCGCTCCGGATGGTGGGGCGGCCAGCGCCGAGGCCGGTCCTGGGACCAGGAC 120 CGGGCGGTGCGCTCCGGATGGTGGGGCGGCCAGCGCCGAGGCCGGTCCTGGGACCAGGAC 120

$\begin{array}{llllllllllllllllllll}R & K & \text { W } & \text { F } & \text { E } & \text { T } & \text { W } & \text { D } & \text { E } & \text { E } & \text { V } & \text { V } & \text { G } & \text { T } & \text { F } & \text { S } & \text { N } & \text { W } & \text { G } & \text { F }\end{array}$ MUC3A_CT AGGAAATGGTTCGAGACCTGGGATGAGGAAGTCGTGGGCACTTTTTCAAACTGGGGTTTC 180 MUC3B_CT AGGAAATGGTTCGAGACCTGGGATGAGGAAGTCGTGGGCACTTTTTCAAACTGGGGTTTC 180

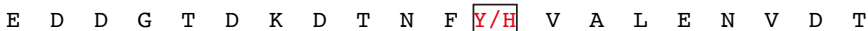
MUC3A CT GAGGACGACGGAACAGACAAGGATACAAATTTCTATGTGGCCTTGGAGAACGTGGACACC 240 MUC3B_CT GAGGACGACGGAACAGACAAGGATACAAATTTCCATGTGGCCTTGGAGAACGTGGACACC 240 $\begin{array}{lllllllllllllllllll}T & M & K & V & H & I & K & R & P & E & M & T & S & S & S & V & *\end{array}$

MUC3A_CT ACTATGAAGGTGCACATCAAGAGACCCGAGATGACCTCGTCCTCAGTGTGA 291 MUC3B_CT ACTATGAAGGTGCACATCAAGAGACCCGAGATGACCTCGTCCTCAGTGTGA 291
\end{abstract}

PDZ binding motif 
Fiqupepgiv preprint doi: https://doi.org/10.1101/2021.12.31.474548; this version posted January 1, 2022. The copyright holder for this preprint (which was not certified by peer review) is the author/funder, who has granted bioRxiv a license to display the preprint in perpetuity. It is

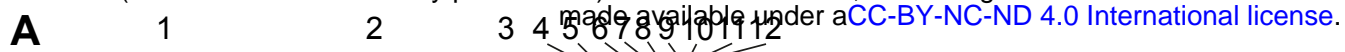

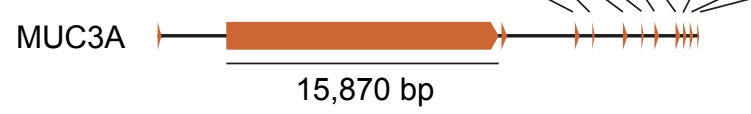

MUC3B

$39,267 \mathrm{bp}$

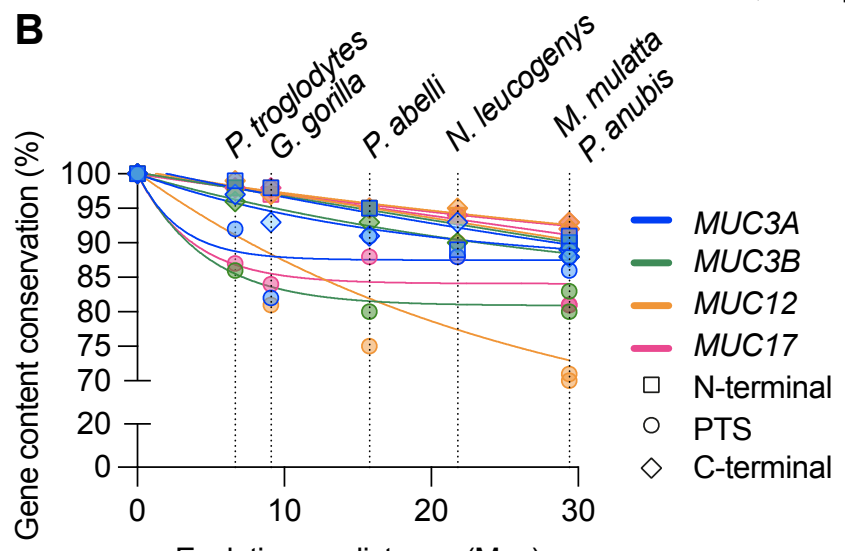

C 0 Evolutionary distance (Mya) 47,759

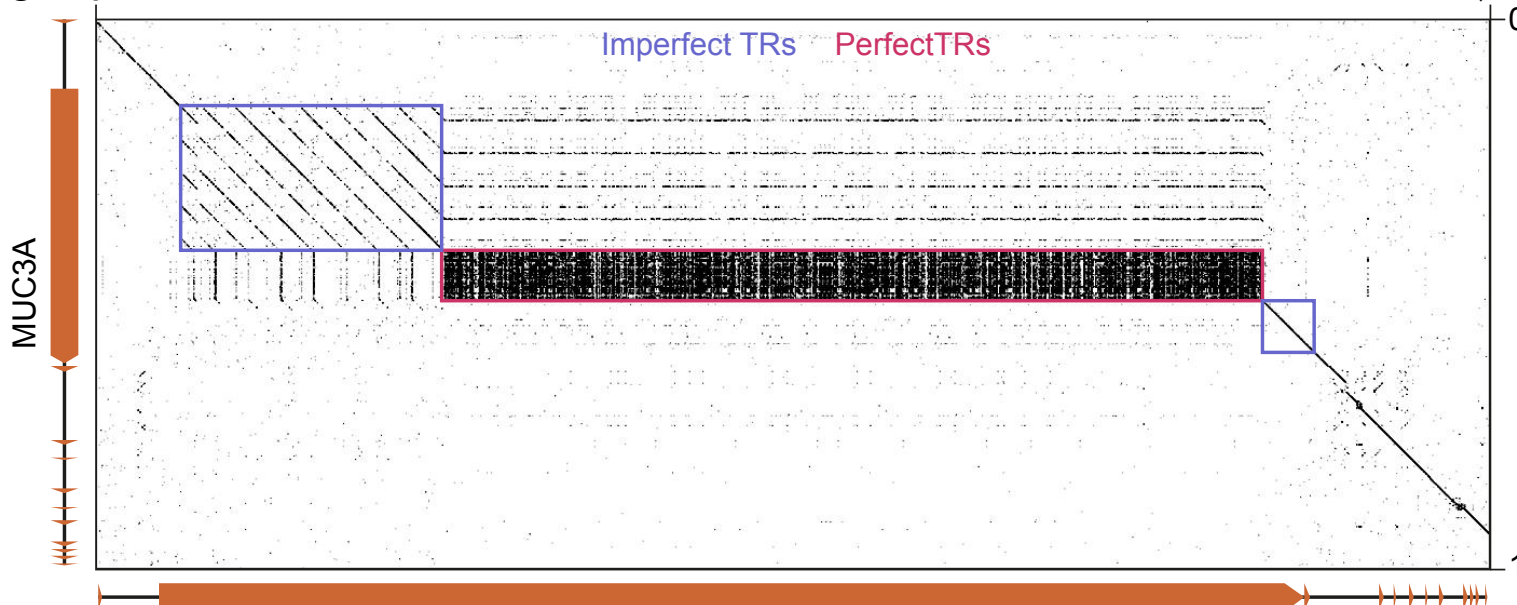

MUC3B
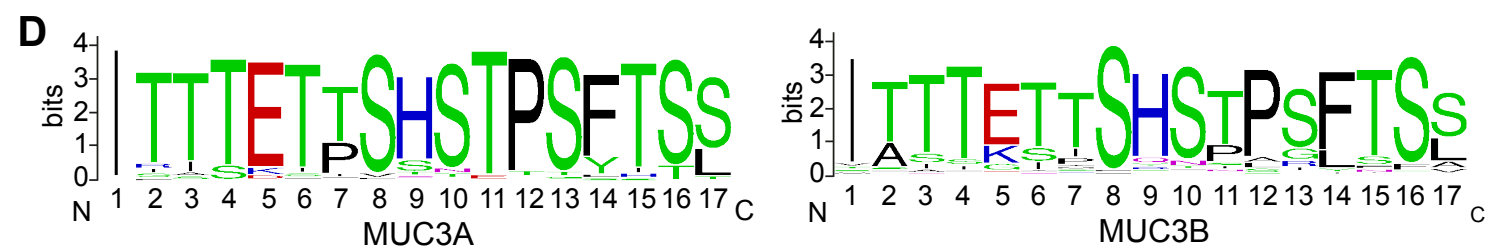
Figupęquiv preprint doi: https://doi.org/10.1101/2021.12.31.474548; this version posted January 1, 2022. The copyright holder for this preprint (Which was not certified by peer review) is the author/funder, who has granted bioRxiv a license to display the preprint in perpetuity. It is A MUC3A Exon 1 made available under aCC-BY-NC-ND 4.0 International license.

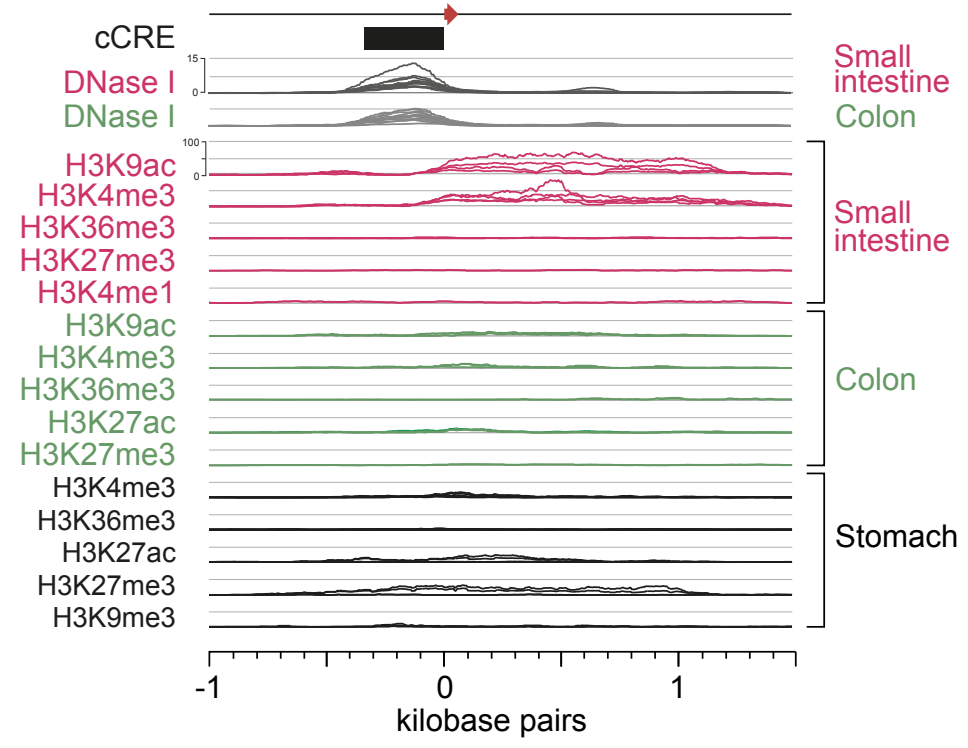

\section{B}

Mouse

\section{Small intestine}

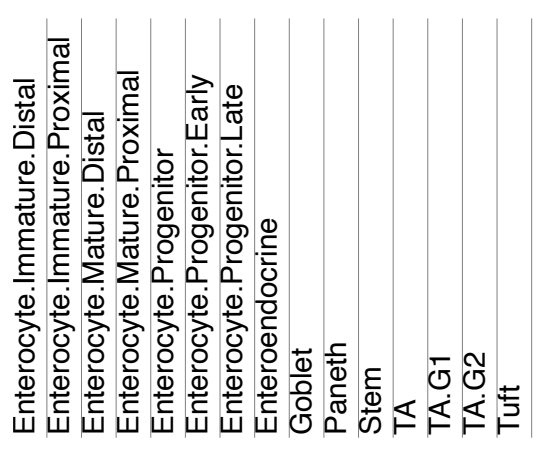

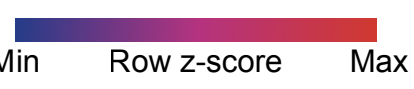

Human

Small intestine
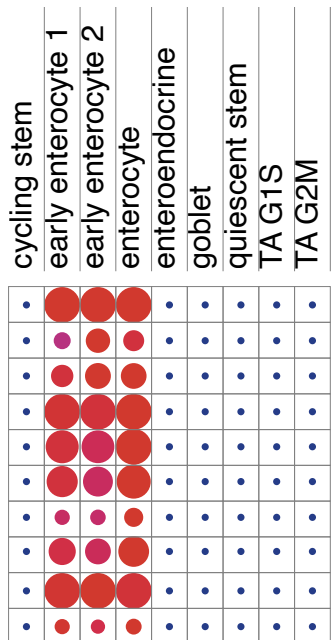

HNF4A

HNF4G

STAT3

ELF3

KLF4

PPARA

RXRA

NR2F6

XBP1

CREB3

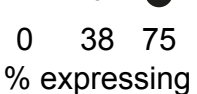

$\%$ expressing

Human

Colon

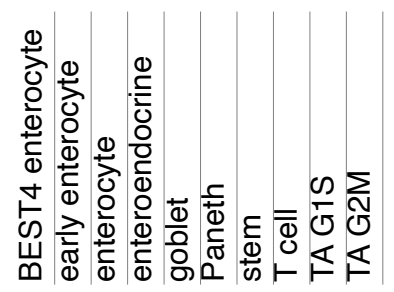

C

STAT3

cCRE

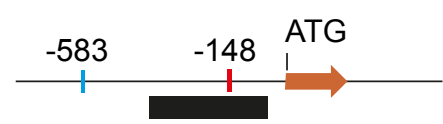

\section{HNF4A/HNF4G}

HOMMUC3A GCCCCAACTCTTCAGATAATAAAATGACTTCAGATGGAATTCTGTGTTAA -542 PanMUC3A GCCCCAACTCTTCAGATAATAAAATGACTTCAGATGGAATTCTGTGTTAA GorMUC3A GCCCCAACTCTTCAGATAATAAAATGACTTCAGATGGAATTCTGTGTTAA PonMUC3A GCCCCAACTCTTCAGATAATAAAATGACTTCAGATGGAATTCTGTGTTAA MaCMUC 3A GCCCCAACTCTTCAGATAATAAATGACTTCAGACGGAATTCTGTGTTAA DOMUC3A GCCCCAACTCTCDCATAATAAATCACTCAGACGAAT

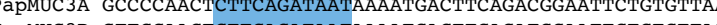
HOMUUC3B GICCAACT PONMUC3B GTMCCAACICTTCAGATAATAAAATGACTTCAGATGGAATTCTGTGTTAA PONMUC3B GTCCCAACTCTTCAGATAATAAAATGACTTCAGATGGAGTTCTGTGTTAA MacMUC3B GCCCCAACTCTTCAGATAATAAAATGACTTCAGACGGAATTCTGTGTTAA PapMUC3B GCCCCAACTCTTCAGATAATAAAATGACTTCAGACGGAATTCTGTGTTAA

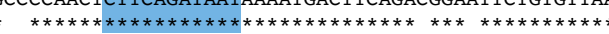

CGGGTGCAGGAGAAGGGAGAAGAGGGGAGAGTGGGGCGTGAAAGTGAGGCTGTG -127 CGGGTGCAGGGAGAAGGGAGGAAGAGGGGAGAGGTGGGGCGGTGCAAAGGTGAGGCTGTG CGGGTGCAGGGAGAAGGGAGGAAGAGGGGAGAGGTGGGGCGGTGCAAAGGTGAGGCTGTC CGGGTGCAGGGAGAGGGAGAAGAGGGAAAGTGGGGCGGTGCAAAGGTGAGGCTGTG CGGATGCACGGACAAGCGACGAAAGCGTTGACGTGCCCCGGTGCAAAGGTGACGCTCCG CGG (1) CAGATGCAGGGAGAAGGAGAAAAGGGTAGGTGGGCGGTGAAAGGTGAGGCTGTG CAGATGCAGGGAGAAGGGAGGAAAAGGGGTGAGGTGGGGCGGTGCAAAGGTGAGGCTGT CAGATGCAGGGAGAAGGGAGGAAATGGGGTGAGGTGGGGCGGTGCAAAGGTGAGGCTGT CAGATGCAGAGAGAAGGGAGGAAGAGGGGTGAGGTGGGGCGGTGCAAAGTTGAGGCTGTC CAGATGCAGAGAGAAGGGAGGAAGAGGGTTGAGGTGGGGGGTGCAAAGGTGAGGCTGTG $* * * * * * \quad * * * * * * * * * * * * \quad * * * * * * * * * * * * * * * * * * * * * * * * * * * * * * *$ 
FiqupiegFiv preprint doi: https://doi.org/10.1101/2021.12.31.474548; this version posted January 1, 2022. The copyright holder for this preprint (which was not certified by peer review) is the author/funder, who has granted bioRxiv a license to display the preprint in perpetuity. It is

A

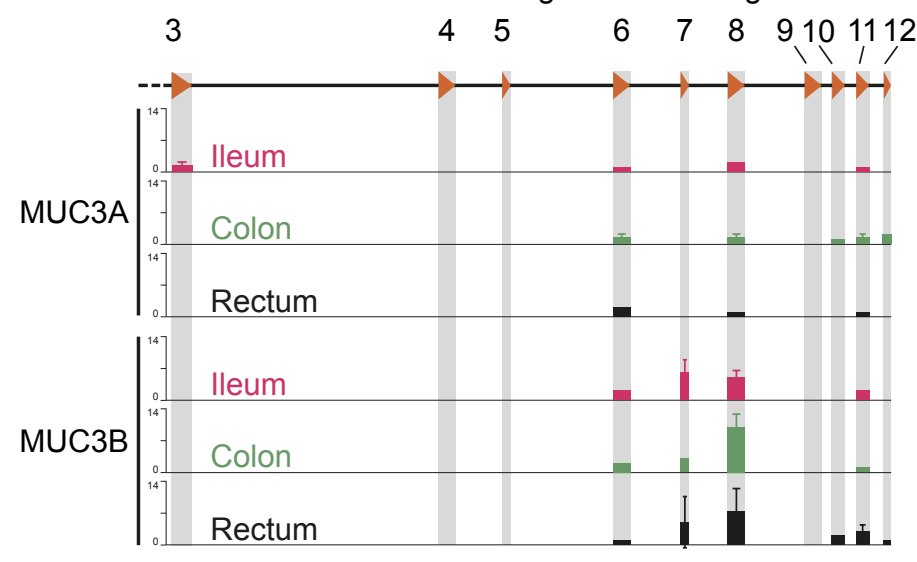

Gene-specific reads
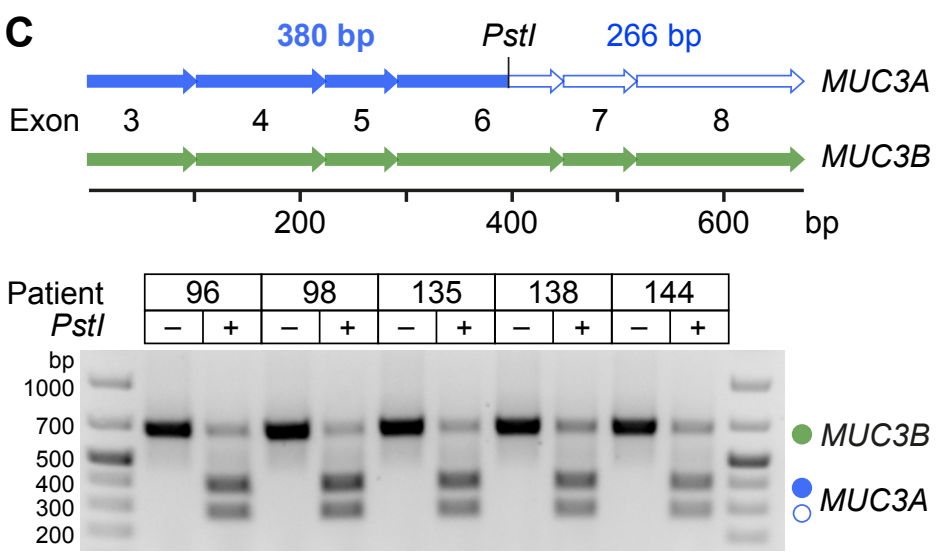

MUC3A-specific primers

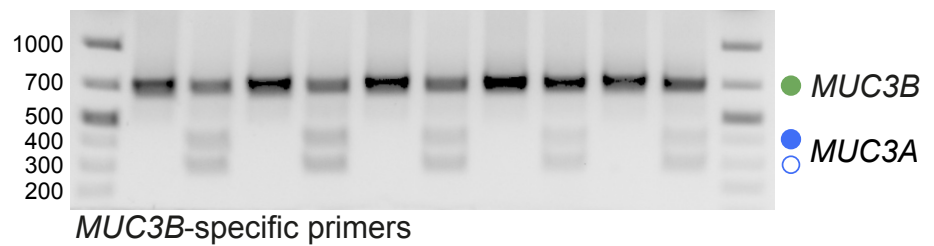

MUC3B-specific primers

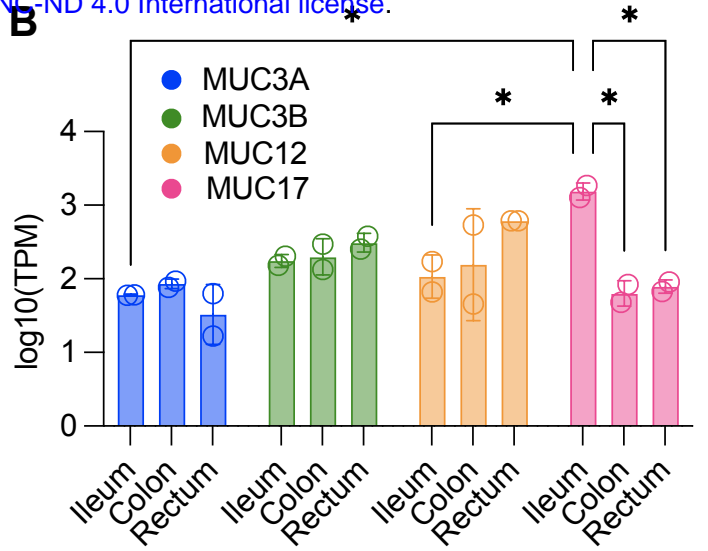

D

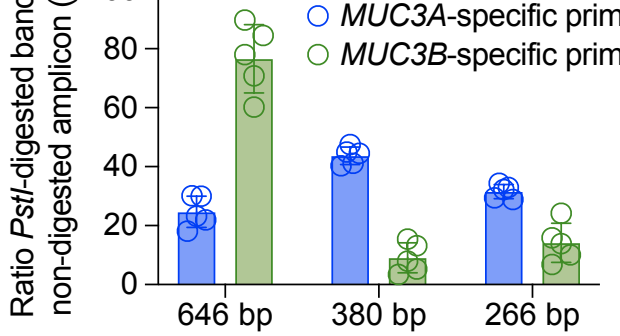

